\title{
The impact of a multicomponent-functional training with postural correction on functional balance in the elderly with a history of falling
}

Parisa Sedaghati ${ }^{*}$, Maryam Goudarzian ${ }^{2 *}$, Somayeh Ahmadabadi ${ }^{3}$ and Seyed Mojtaba Tabatabai-Asl ${ }^{1}$

\begin{abstract}
Purpose: Good posture plays a significant role for the elderly in achieving optimal quality of life. This study aimed to evaluate the impact of multicomponent functional training with postural correction on functional balance in the elderly with a history of falling.

Methods: This study was a randomized controlled, single-blind study. Subjects ( $n=28$, mean age $=70$ years) with a history of falling were selected and randomly allocated to either a multicomponent functional training $(n=14)$ or a control group $(n=14)$. The experimental group exercised for 8 weeks, three days per week for 60 min per day. The training program with strength, endurance, and balance parts was conducted in the multi-task conditions to stimulate the physical and cognitive abilities focusing on the attentional-correct posture. The control group received conventional care. The Berg balance and short physical performance battery tests were used in the pre-test and post-test. The adjusted post-test means of experimental and control groups were analyzed using the ANCOVA test to eliminate any pretest effects.
\end{abstract}

Results: This study found a significant effect of training on Berg balance test $(P=0.001)$, Timed Up and Go with D-T $(P=0.01)$, Timed Up and Go $(P=0.002)$, and Short Physical Performance Battery $(P=0.001)$.

Conclusions: Eight weeks of multicomponent exercise training has beneficial effects on balance and physical function and results in improved equilibrium and a decreasing probability of falling. Therefore, practitioners can use this 8-week training program for older adults.

Keywords: Aging, Fall, Intervention, Physical activity

\footnotetext{
*Correspondence: sedaghati@guilan.ac.ir; goudarzianmaryam@gmail.com

${ }^{1}$ Department of Sports Injuries and Corrective Exercise, Faculty

of Physical Education and Sport Sciences, University of Guilan, Rasht, IR,

Iran

${ }^{2}$ Iranian Research Center On Healthy Aging, Sabzevar University

of Medical Sciences, Sabzevar, IR, Iran

Full list of author information is available at the end of the article
} 


\section{Introduction}

One-third of the elderly with age over 65 years living in urban experience falls each year in China [12]. Falls account for over $80 \%$ of hospitalizations for patients aged 65 years or over [26]. Several risk factors for falling include balance impairment, decreased muscle strength, and gait impairment [44]. Interventions that address multiple risk factors have demonstrated a significant reduction in falls among community-dwelling older adults [21].

Reduced mobility is considered one of the main predictors of falling in older adults [8]. Hence, it seems very important to introduce a fall prevention program that targets mobility for such age groups, especially for those living in nursing and residential care facilities [30]. Otherwise, the percentage of older people staying in nursing homes would also significantly increase. In care homes, older adults constitute a diverse and heterogeneous community with a high prevalence of dependence in everyday life tasks, cognitive disability, depression, a high fall incidence, multi-morbidity, and poly-medication [15]. Besides, old people living in nursing homes for a long time often tend to be highly inactive, participating in sedentary activities for most of the day [5].

The cause of falls is multifactorial, with one major factor being compromised postural function. Postural control is the ability to control the body position in space during standing and walking tasks for stabilization and orientation purposes [39].

Dual-task efficiency refers to the ability to execute two tasks concurrently with the postural double-task management relating to situations when the postural control requires at least one of the tasks, such as walking while talking on the phone, or carrying a bowl or cup [40]. Deficiency in dual-task postural control is associated with decreased cognitive performance in older adults [25] and an elevated rate of falls in the elderly [32]. Some everyday life activities are multitasking activities that generate conflicting demands on attention and involve challenging tasks and cognitive functions [34]. While attention is limited and demands are higher than capacity, a dual-task performance might be affected in a single-task performance compared to the performance of the same tasks [2]. For accomplishing daily activities independently, altered management of attention capital is considered [42]. For evaluating this relationship, much research has focused on the interaction between postural control and using the dual-task postural control paradigms [6].

In recent years, many studies have shown that old age is no deterrent to improving coordination, power, and autonomous transfer by introducing adapted physical activity [13]. In a systematic and meta-analysis, findings showed that various balance training modalities lead to changes in static/dynamic steady-state, constructive, and reactive balance assessments as well as in the efficiency of balance test batteries in stable older people [28]. A successful balance training program for healthy older adults consists of 11-12 weeks, three sessions per week for a total of 36-40 sessions, 31-45 min per session, and 91-120 min of balance training per week [28]. While Agmon et al. indicated prospective approaches to strengthen postural control, dual-task management should provide centered dual-task preparation and resolve activities that are more associated with fall risk [1]. Therefore, Agmon et al. stated for evaluating the best appropriate protocol, prospective studies may also concentrate on motor learning features that can extend the retention of dual-task training benefits [1]. The novelty of this study refers to the multi-component functional training (MCFT) with components of functional balance and lower-extremity muscle strength affecting postural correction during physical activity and also functional balance assessment in single-task and dual-task conditions.

Therefore, this study aimed to evaluate the impact of multicomponent-functional training with postural correction on functional balance and the probability of falling in the elderly living in nursing homes with a history of falling.

\section{Materials and methods \\ Participants}

Participants included 28 males recruited from Elderly Care Center $\quad$ age $=70.8 \pm 2.5$ years, Weight $=66.6 \pm 2.3 \mathrm{~kg}$, height $=167.9 \pm 2.4 \mathrm{~cm}, \mathrm{BMI}=23.6 \pm 1.1$ ). Participants were randomly allocated to either multicomponent functional training (MCFT) or control group (CG). All the participants completed and signed informed consent, and all ethical considerations were observed based on the Helsinki Declaration. Figure 1 shows the CONSORT flowchart of the study and the allocation procedure of subjects to groups. Also, the reporting checklist for randomized trials based on CONSORT guidelines can be found in Appendix 1.

\section{Procedure}

In this randomized-controlled study, the inclusion criteria were as follows: 1 ) age $\geq 68$ years old, 2) Farsi Version of the Mini-Mental State Examination (MMSE) $>24$ $[20], 3)$ walking or accomplishing other daily activities without assistance, 4) being healthy without any acute or chronic diseases or physical, mental, psychological, and other disorders (e.g., cardiovascular, respiratory, skin problems, osteoarthritis), 5) the ability to participate in 


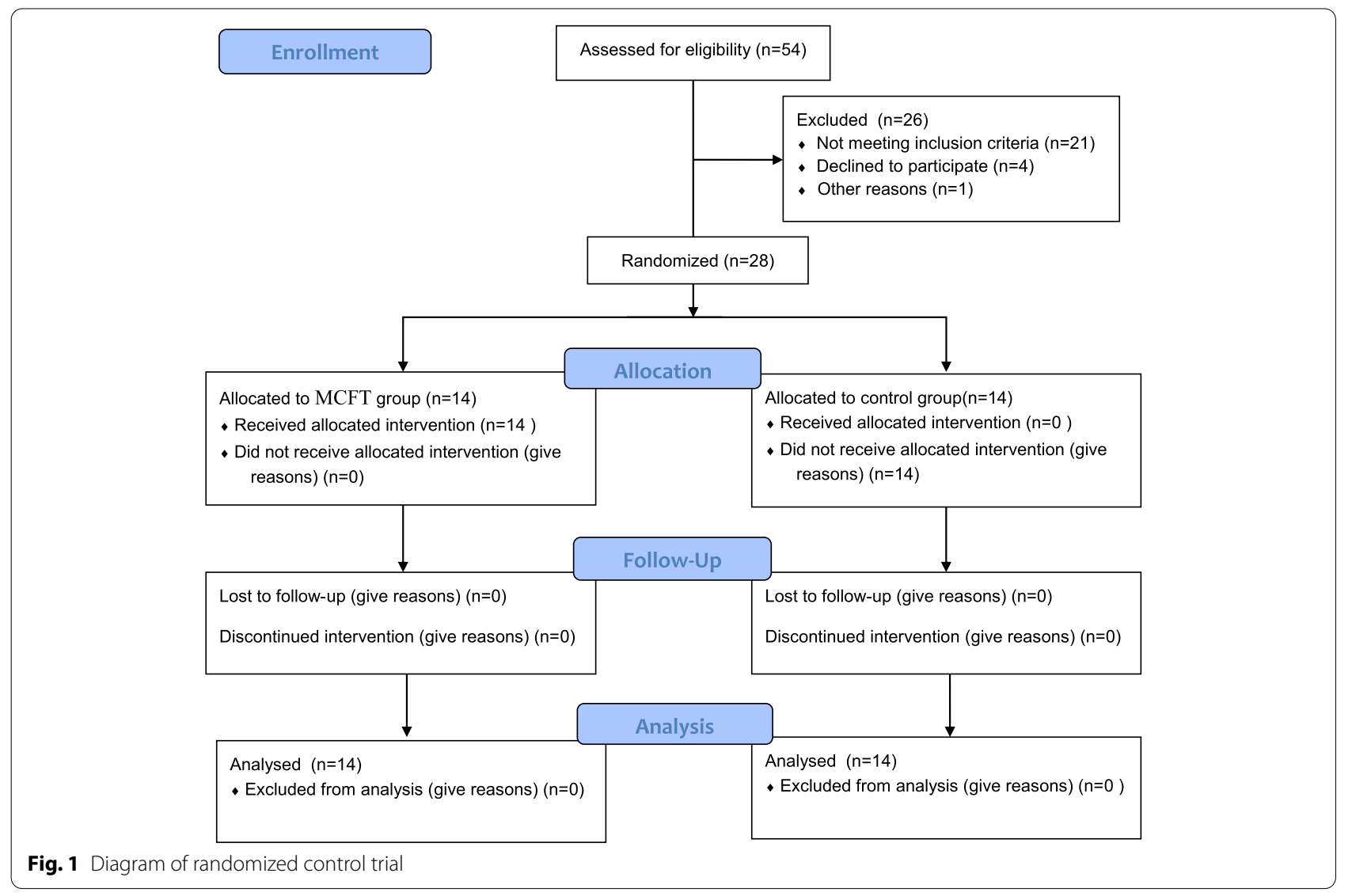

regular training sessions (not missing more than two sessions during the entire research period), 6) no history of regular physical activity in the 6 months before participating in the study, 7) the ability to stand for at least one minute and walk $10 \mathrm{~m}$ unaided, 8 ) normal vision (or corrected vision).

Exclusion criteria were as follows: 1) a history of depression or other psychological disorders, 2) orthopedic problems or severe lower/upper extremity deformities, 3) a history of lower limb joint replacement, 4) a history of balance disorders and recurrent positional vertigo, 5) severe pain in lower/upper extremities, 6) vestibular diseases, 7) a severe visual impairment, 8) unwillingness to continue participation in the study, 9) missing three consecutive training sessions. Participants were not eligible whenever the medical staff realized they were clinically unstable or showed no inclination to continue the study [1, 35, 38]. All participants were informed of the purpose and possible risks involved in the research study and were required to read and sign an informed consent form before participation. sand the study was registered in the Iranian Registry of Clinical Trials (IRCT20160815029373N5).

\section{Randomization}

After the primary assessment, the participants will be randomly (lottery method) allocated into either MCFT or CG group in a double-blinded design for both the participants and assessors.

\section{Exercise intervention \\ MCFT}

Practicability of the MCFT were assessed by a pilot study with full details of volume, intensity, and the type of strength and balance exercises using the dual-task training $[1,4,33,35,36]$. Participants allocated to this MCFT group were attended a training program, three times a week for an hour per session. The program consisted of strength and balance exercises performed and thought by an experienced exercise physiologist. This point should be mentioned that participants must maintain correct 
Table 1 MCFT Program for the 8 weeks and progression of the complexity of secondary tasks $[4,33]$

\begin{tabular}{|c|c|c|}
\hline Phases program & First month & Second month \\
\hline Type of physical activity & $\begin{array}{l}\text { Strength Static balance }+ \text { dual-task }+ \text { corrective } \\
\text { posture }^{a}\end{array}$ & $\begin{array}{l}\text { Strength }+ \text { Dynamic balance }+ \text { dual-task }+ \text { cor- } \\
\text { rective posture }^{a}\end{array}$ \\
\hline 1-Warm-up 5 min & Range of motion for different joints & \\
\hline $\begin{array}{l}\text { 2-Strength lower extremity: Chair squat and } \\
\text { stand, Leg flexion, Leg extension, Leg abduction, } \\
\text { Hip extension, Standing on the tips of toes and } \\
\text { heels }\end{array}$ & 4-5 ex: 2 sets, 8 rep & 4-5 ex:1-2 sets, 12 rep \\
\hline $\begin{array}{l}\text { 3-Balance: Feet-together stance, legged stand, } \\
\text { Semi-tandem/Tandem balance, Circuit training, } \\
\text { Gait training-stairs \& obstacle function }\end{array}$ & $\begin{array}{l}2-3 \text { ex, progressive difficulty in sitting position } \\
\text { and progressing to standing position }\end{array}$ & $\begin{array}{l}\text { 4-5 ex, progressive difficulty in standing position } \\
\text { with decreasing arm support and increasing } \\
\text { instability with foam mat }\end{array}$ \\
\hline $\begin{array}{l}\text { 4- Dual-task (cognitive function): One type of } \\
\text { cognitive exercise in each session) Naming } \\
\text { colors/days of the week/names, Counting by } \\
\text { twos starting } 0 \text { till a number } \leq 30 \text {, Counting } \\
\text { backward by ones( }\end{array}$ & In 2-3 of strength ex and 1-2 of balance ex & In $1-2$ of strength ex and 3-4 of balance ex \\
\hline 5-Cool down 5 min & Stretching, breathing exercises & \\
\hline
\end{tabular}

ex exercises, rep repetitions

a Participants must maintain correct posture during all types of physical activity

Anthropometric measurements

Outcome Variable

Table 2 Tests to assess the functional abilities

\begin{tabular}{|c|c|c|}
\hline Test & Parameters & Description \\
\hline Berg balance test & Postural stability & $\begin{array}{l}\text { Performance of } 14 \text { functional tasks [7]. The inter-rater reliability } \\
\text { was also high, with a pooled estimate of } 0.97[17]\end{array}$ \\
\hline Timed Up and Go (with and without D-T) & Functional balance & $\begin{array}{l}\text { Get up from a chair, walk } 3 \mathrm{~m} \text { at a normal pace, turn around, } \\
\text { walk back to the chair and sit down again }[31,35] \text {, participant } \\
\text { test was carried out by carrying a ball }[1,14] \text {. The test-retest } \\
\text { reliability (ICC) ranged from } 0.54 \text { to } 0.85[11]\end{array}$ \\
\hline Short Physical Performance Battery & $\begin{array}{l}\text { Lower extremity function: static balance, } \\
\text { gait speed, and getting in and out of a } \\
\text { chair }\end{array}$ & $\begin{array}{l}\text { Side-by-side, semi-tandem, and tandem stands }(10 \mathrm{~s}) ; 4 \mathrm{~m} \text { walk } \\
\text { test at a comfortable speed and } 5 \text { quickly sit to stand from a } \\
\text { chair without upper extremity assistance [1,24]. test-retest } \\
\text { reliability of the SPPB was high: } 0.87 \text { for subjects aged } 65 \text { to } \\
74 \text { years[23] }\end{array}$ \\
\hline tandem gait test(TGT) & Dynamic gait balance & $\begin{array}{l}\text { to take } 12 \text { consecutive steps with the feet aligned heel to toe } \\
\text { in tandem on a straight line with their eyes open (stopwatch), } \\
\text { without walking aids, and with their arms hanging by the sides } \\
\text { of their body [27]. Reliability coefficients were } 0.62 \text { for tandem } \\
\text { gait for the disabled sample[22] }\end{array}$ \\
\hline
\end{tabular}

posture during all types of physical activity. Also, participants continue their daily activities as usual.

In the multicomponent functional training (MCFT), physical activities were designed based on executive functions and conducted alongside approximately four MCFT. The difficulty of dual-tasks increased by augmenting the complexity of motor tasks mentioned in Table1.

The primary outcome measures presented in Table 2 consist of Short Physical Performance Battery (SPPB), Berg balance test (BBT), Timed Up and Go (with and without D-T), tandem gait test, and anthropometric measurements: weight, height, body mass index [18]. Height and weight were measured to the nearest $0.1 \mathrm{~cm}$ and $0.1 \mathrm{~kg}$, respectively.

\section{Power and sample size}

The sample size was calculated using $G$ * Power software which evaluated an exercise program with TUG performance of 28 subjects, with a mean time to perform TUG before and after the intervention of $17.92 \pm 9.42$ 
Table 3 Comparing the demographic data of the participants between the experimental and control groups

\begin{tabular}{lccc}
\hline Group & $\begin{array}{c}\text { EG }(\boldsymbol{n}=\mathbf{1 4}, \mathrm{F}=\mathbf{7} ; \mathrm{M}=\mathbf{7}) \\
\text { Mean } \pm \text { SD (range) }\end{array}$ & $\begin{array}{l}\text { CG }(\boldsymbol{n}=\mathbf{1 4}, \mathrm{F}=\mathbf{7} ; \mathbf{M}=\mathbf{7}) \\
\text { Mean } \pm \text { SD (range) }\end{array}$ & $\boldsymbol{P}$ \\
\hline Age (y) & $70.42 \pm 2.70$ & $71.07 \pm 2.26$ & $\mathrm{NS}$ \\
Height (cm) & $168.42 \pm 2.40$ & $167.35 \pm 2.23$ & $\mathrm{NS}$ \\
Weight (kg) & $67.12 \pm 5.19$ & $66.05 \pm 2.37$ & $\mathrm{NS}$ \\
BMl & $23.68 \pm 1.21$ & $23.59 \pm 1.11$ & $\mathrm{NS}$ \\
MMSE (score) & $26.08 \pm 1.25$ & $26.33 \pm 1.37$ & $\mathrm{NS}$ \\
Fall number & $2.62 \pm 0.50$ & $2.69 \pm 0.48$ & $\mathrm{NS}$
\end{tabular}

EG Experimental Group, CG Control Group, NS non-significant, MMSE MiniMental State Examination

and $16.35 \pm 7.55 \mathrm{~s}$, respectively. Given a $P$-value of 0.05 , a power of $80 \%$ [3], the TUG test time in seconds, and an effect size of 0.7 for the minimal clinically significant differences, 14 volunteers would be needed in each group (Fig. 1).

\section{Statistical analysis}

Statistical analysis was performed using the software SPSS version 16. For assessing normality of distribution and comparison of demographic variables, the Shapiro-Wilk test, and the independent sample t-test were performed respectively. For excluding any possible pre-test effect, the adjusted post-test means were tested using ANCOVA and for comparison of the means of within-group between the pre-/post-test, the paired t-test was used. The level of significance was fixed at 0.05 and 0.01 level.

\section{Results}

The general demographic characteristics of the 28 subjects are showen in Table 3 and there were no significant differences between groups in examined variables $(P>0.01)$. The independent $\mathrm{t}$-test revealed no significant difference at pre-test in the demographic data between the two groups $(P>0.01)$.
Table 5 Analysis of Covariance for the Selected Variables among Experimental Group \& Control Groups $(n=28)$

\begin{tabular}{lcrll}
\hline Variables & $\begin{array}{l}\text { Type III Sum of } \\
\text { Squares }\end{array}$ & $\mathbf{F}$ & Sig & $\begin{array}{l}\text { Partial Eta } \\
\text { Squared }\end{array}$ \\
\hline BBS & 87.87 & 35.08 & $0.001^{\mathrm{a}}$ & 0.626 \\
TUG(S) & 3.38 & 6.62 & 0.018 & 0.240 \\
TUG-Dual(s) & 1.69 & 8.09 & $0.01^{\mathrm{a}}$ & 0.278 \\
TG & 8.11 & 12.69 & $0.002^{\mathrm{a}}$ & 0.377 \\
SPPB & 7.62 & 14.72 & $0.001^{\mathrm{a}}$ & 0.412 \\
\hline
\end{tabular}

BBS Berg Balance Scale, TUG Timed Up and Go test, TG tandem gait, SPPB Short Physical Performance Battery test, ${ }^{\text {a }}$ Statistically significant.

As shown in Table 4. the paired t-test of BBS, TUG, TUG-D, TG, and SPPB in the control group revealed no significant difference between the pre-/post-test $(P \geq 0.01)$. However, the paired $\mathrm{t}$-test showed a significant difference between the pre-/post-test $(P=0.001)$ for the effects of MCFT on BBS, TUG, TUG-D, TG, and SPPB.

As shown in Table 5, the ANCOVA test with covariate pre-test revealed a significant difference in BBS $(P=0.001)$, TUG $(P=0.018)$, TUG-D $(P=0.01)$, TG $(P=0.002)$, and SPPB $(P=0.001)$ between post-test of experimental and control groups.

\section{Discussion}

The current study explored the effect of a MCFT program on balancing single- and dual-tasks, including gait and cognitive tasks, in elderly subjects with a history of falling while residing in nursing homes.

This study found a significant effect of MCFT on BBS, TUG, TUG-D, TG, and SPPB between the pre-/post-test and the exercise and control groups.

Considering the exponentially rising number of people over 68 , there is a lack of recommendations for the geriatric population and practitioners working in this sector. Older adults are at specific risk of adverse effects

Table 4 The Pre and Post Test Means of Experimental and Control Groups $(n=28)$

\begin{tabular}{|c|c|c|c|c|c|c|c|c|}
\hline \multirow[t]{2}{*}{ Groups } & \multicolumn{4}{|l|}{$\mathrm{E} \mathrm{G}(n=14)$} & \multicolumn{4}{|l|}{$C G(n=14)$} \\
\hline & PreT & PostT & $\mathrm{T}$ & $P$ & PreT & PostT & $\mathbf{T}$ & $P$ \\
\hline BBS & $27.00 \pm 2.18$ & $31.00 \pm 3.13$ & -7.95 & $0.001^{a}$ & $26.00 \pm 1.79$ & $25.64 \pm 1.90$ & 0.92 & 0.373 \\
\hline TUG(s) & $15.11 \pm 1.36$ & $13.87 \pm 1.64$ & 5.54 & $0.001^{a}$ & $14.86 \pm 1.60$ & $15.05 \pm 1.60$ & -1.24 & 0.236 \\
\hline TUG-Dual(s) & $16.33 \pm 1.47$ & $15.39 \pm 1.67$ & 5.94 & $0.001^{a}$ & $16.32 \pm 1.60$ & $16.37 \pm 1.62$ & -0.61 & 0.548 \\
\hline TG & $6.35 \pm 1.00$ & $8.00 \pm 1.24$ & -8.25 & $0.001^{a}$ & $6.14 \pm 1.23$ & $6.50 \pm 1.09$ & -0.36 & 0.720 \\
\hline SPPB & $6.42 \pm 0.85$ & $8.21 \pm 1.25$ & -8.33 & $0.001^{a}$ & $6.28 \pm 1.20$ & $6.35 \pm 1.15$ & -1.58 & 0.136 \\
\hline
\end{tabular}

EG Experimental Group, CG Control Group, PreT Pre Test, PostT Post Test, BBS Berg Balance Scale, TUG Timed Up and Go test, TG tandem gait, SPPB Short Physical Performance Battery test, ${ }^{\text {aS }}$ tatistically significant 
in long-term nursing homes where they have been the subject of strategies to avoid or reverse frailty [46].

Dual-task training was introduced as functional training focusing on physical parameters and cognitive variables in the elderly $[19,29]$. Dual-task paradigms were widely used to evaluate the degree of automatic and controlled handling of postural stability among different age groups [10]. The present study used a previously published protocol on dual-tasks which was applicable and it showed improvements in many functional outcomes [19, 29, 35, 37]. Besides, the proposed interventions are easy to apply and include extensive practical issues on application (e,g., training frequency, volume, intensity, individualization, and rest intervals). These training regimes are easy to use in long-term nursing homes. Thus, current findings provide valuable insight into the impact of the dual-task program on patients living in long-term in a nursing home that integrates physical and cognitive factors consistent with aging. Moreover, analysis of an MCFT program and the same program with concurrent cognitive training, or dual-task, supported us to design the interventions to improve or at least maintain functionality and cognition in long-term nursing home peoples. In this regard, Rezola-Pardo et al. reported the effects of the dual-task program on people living in long-term in a nursing home, taking overall physical, cognitive, and emotional variables linked to frailty [35]. Furthermore, the analysis of an MCFT program and the same program with concurrent postural training, dual-task, supported our findings to design the interventions to improve or maybe maintain functionality in nursing home peoples [35].

Comparison our findings with recently published articles on young healthy adults, it seems that the effect of dual-task protocols is independent of age [28]. Given the limited number of training protocols [28], further investigation is necessary to prove and specify preliminary dosage-responding relationships of dual-task physical training protocols in healthy older adults.

Critically, the review by Zijlsra et al. (2008) questioned the added value of dual over single postural task conditions for fall prediction [47]. Stins and Beeks (2012) also expressed reservations about the possibility for the cognitive processes to influence postural control [41]. Moreover, Agmon et al. reported no improvement in transfer between single-task and dual-task performance [1].

Most studies evaluating postural performance in the static standing revealed a difference in performance between healthy older adults and young adults [45] that may be related to a greater incidence of falls in older adults as an indicator of declined postural stability [9].

On the other side, considering a relation between dual-task training and fall, Agmon et al. suggested that future studies should focus on dual-task training and report tasks that have the highest correlation with the risk of falling to improve dual-task postural control. Moreover, a long-term follow-up of fall occurrences and daily activity should apply to provide a better understanding of whether improved dual-task postural control impacts these factors [1]. Furthermore, future research should focus on motor learning elements that may extend the retention of dual-task training benefits to determine the most effective protocols. We believe that several inconsistencies could be resolved by a careful selection of dual-task studies based on the methodological criteria. In line with the improvement in the probability of falling, Tabatabai et al. (2021) studied the impact of combined Cawthorne-Cooksey exercises on functional balance and fall probability in elderly people and their results showed a significant improvement in balance and reduced risk of falling in the adult people [43]. Also, the results of the present study are in line with the study of Długosz et al (2021). They investigated the effects of three months of pilates training on balance and fall risks in older women. Their results showed statistically significant improvements in balance and risks of falling in old people [16].

To the best of our knowledge, no study has explored the impacts of a managed dual-task MCFT in the elderly with a history of falling and living nursing home facilities and assessing functional capacity under both single- and dual-task conditions and physical activity. Moreover, we mention possible limitations of the present study. The selected inclusion criteria excluded the majority of long-term nursing home residents, as we included light to moderately dependent participants, while the prevalent profile in this type of institution is strictly dependent. Therefore, we might come across difficulties in reaching the anticipated sample size. However, the large number of agreements made with long-term care centers with the general office. Welfare Organizations will facilitate the recruitment of sufficient subjects.

\section{Conclusions}

In summary, the present study indicated that a selective posture-corrected multicomponent exercise with a single or dual-task can improve the functional balance in elderly residents in a nursing home with a history of falling. 


\section{Appendix}

\section{Table 6}

Table 6 CONSORT 2010 checklist of information to include when reporting a randomize trial

Section/Topic Item No Checklist item

\section{Title and abstract}

\section{Introduction}

Background and objectives

\section{Methods}

Trial design

Participants

Interventions

Outcomes

Sample size

Randomisation:

Sequence generation

Implementation

Blinding

Statistical methods

\section{Results}

Participant flow (a diagram is strongly recommended)

Recruitment

Baseline data

Numbers analysed

Outcomes and estimation 1b Structured summary of trial design, methods, results, and conclusions (for specific 1 guidance see CONSORT for abstracts)

Scientific background and explanation of rationale Specific objectives or hypotheses

Description of trial design (such as parallel, factorial) including allocation ratio Important changes to methods after trial commencement (such as eligibility criteria), with reasons

Eligibility criteria for participants

The interventions for each group with sufficient details to allow replication, including 5-6 how and when they were actually administered

Completely defined pre-specified primary and secondary outcome measures, 6 including how and when they were assessed

Any changes to trial outcomes after the trial commenced, with reasons How sample size was determined

When applicable, explanation of any interim analyses and stopping guidelines

Mechanism used to implement the random allocation sequence (such as sequentially numbered containers), describing any steps taken to conceal the sequence until interventions were assigned

Who generated the random allocation sequence, who enrolled participants, and 5 who assigned participants to interventions

If done, who was blinded after assignment to interventions (for example, participants, care providers, those assessing outcomes) and how

If relevant, description of the similarity of interventions

Statistical methods used to compare groups for primary and secondary outcomes Methods for additional analyses, such as subgroup analyses and adjusted analyses

For each group, the numbers of participants who were randomly assigned, received 7 intended treatment, and were analysed for the primary outcome

For each group, losses and exclusions after randomisation, together with reasons $\quad 7$ Dates defining the periods of recruitment and follow-up 7-8 Why the trial ended or was stopped $\quad \mathrm{N} / \mathrm{Y}$ A table showing baseline demographic and clinical characteristics for each group $\quad 7-8$ For each group, number of participants (denominator) included in each analysis and 7-8 whether the analysis was by original assigned groups

17a For each primary and secondary outcome, results for each group, and the estimated 7-8 effect size and its precision (such as 95\% confidence interval)

17b For binary outcomes, presentation of both absolute and relative effect sizes is recom- N/Y mended 
Table 6 (continued)

\begin{tabular}{|c|c|c|c|}
\hline Section/Topic & Item No & Checklist item & $\begin{array}{l}\text { Reported } \\
\text { on page } \\
\text { No }\end{array}$ \\
\hline Ancillary analyses & 18 & $\begin{array}{l}\text { Results of any other analyses performed, including subgroup analyses and adjusted } \\
\text { analyses, distinguishing pre-specified from exploratory }\end{array}$ & $N / Y$ \\
\hline Harms & 19 & $\begin{array}{l}\text { All important harms or unintended effects in each group (for specific guidance see } \\
\text { CONSORT for harms) }\end{array}$ & $N / Y$ \\
\hline \multicolumn{4}{|l|}{ Discussion } \\
\hline Limitations & 20 & $\begin{array}{l}\text { Trial limitations, addressing sources of potential bias, imprecision, and, if relevant, } \\
\text { multiplicity of analyses }\end{array}$ & 10 \\
\hline Generalisability & 21 & Generalisability (external validity, applicability) of the trial findings & 8 \\
\hline Interpretation & 22 & $\begin{array}{l}\text { Interpretation consistent with results, balancing benefits and harms, and considering } \\
\text { other relevant evidence }\end{array}$ & $8-10$ \\
\hline Other information & & & $9-10$ \\
\hline Registration & 23 & Registration number and name of trial registry & 12 \\
\hline Protocol & 24 & Where the full trial protocol can be accessed, if available & 6 \\
\hline Funding & 25 & Sources of funding and other support (such as supply of drugs), role of funders & 12 \\
\hline
\end{tabular}

\section{Abbreviations}

Ex: Exercises; Rep: Repetitions; NS: Non-significant; MMSE: Mini-Mental State Examination; EG: Experimental Group; CG: Control Group; PreT: Pre Test; PostT: Post Test; BBS: Berg Balance Scale; TUG: Timed Up and Go test; TG: Tandem gait; SPPB: Short Physical Performance Battery test.

\section{Acknowledgements}

The authors gratefully thank all volunteers who participated and cooperated in this study.

\section{Informed consent}

All the participants completed and signed informed consent, and all ethical considerations were observed according to the Helsinki Declaration.

\section{Authors' contributions}

PS was a major contributor in writing the manuscript. MG helped in the analysis of data. SA helped in writing the manuscript. SMT helped in methodology. The authors read and approved the final manuscript.

\section{Funding}

This work was supported by the Sabzevar University of Medical Sciences under Grant [(IR.MEDSAB.REC.1398.045].

\section{Availability of data and materials}

All raw data sets associated with the paper will be available on request.

\section{Declarations}

\section{Ethics approval and consent to participate}

All procedures performed in studies involving human participants were under the ethical standards of the institutional and national research committee and with the 1964 Helsinki declaration and its later amendments or comparable ethical standards.

\section{Competing interests}

The authors declare that they have no competing interests.

\section{Consent for publication}

Not applicable.

\section{Author details}

${ }^{1}$ Department of Sports Injuries and Corrective Exercise, Faculty of Physical Education and Sport Sciences, University of Guilan, Rasht, IR, Iran. ${ }^{2}$ Iranian Research Center On Healthy Aging, Sabzevar University of Medical Sciences,
Sabzevar, IR, Iran. ${ }^{3}$ Department of Physical Education and Sports Sciences, Farhangian University, Tehran, IR, Iran.

Received: 13 November 2021 Accepted: 16 February 2022

Published online: 01 March 2022

\section{References}

1. Agmon M, Belza B, Nguyen HQ, Logsdon RG, Kelly VE (2014) A systematic review of interventions conducted in clinical or community settings to improve dual-task postural control in older adults. Clin Interv Aging 9:477

2. Albinet $P$, Bernard $P$, Palut $Y$ (2006) Attentional control of postural stability in institutionalised elderly people: effects of a physical exercise program. Ann Readapt Med Phys 49:625-631

3. Alfieri FM, Riberto M, Abril-Carreres À, Boldó-Alcaine M, Rusca-Castellet E, Garreta-Figuera R et al (2012) Effectiveness of an exercise program on postural control in frail older adults. Clin Interv Aging 7:593

4. Arrieta H, Rezola-Pardo C, Zarrazquin I, Echeverria I, Yanguas JJ, Iturburu Met al (2018) A multicomponent exercise program improves physical function in long-term nursing home residents: a randomized controlled trial. Exp Gerontol 103:94-100

5. Bates-Jensen BM, Alessi CA, Cadogan M, Levy-Storms L, Jorge J, Yoshii J et al (2004) The Minimum Data Set bedfast quality indicator: differences among nursing homes. Nurs Res 53:260-272

6. Beauchet $\mathrm{O}$, Annweiler C, Dubost V, Allali G, Kressig R, Bridenbaugh S et al (2009) Stops walking when talking: a predictor of falls in older adults? Eur J Neurol 16:786-795

7. Berg K, Wood-Dauphinee S, Williams J, Maki B (1992) Measuring balance in the elderly: validation of an instrument. Can J Public Health 83(Suppl 2):S7-11

8. Bergland A, Jarnlo G, Laake K (2003) Predictors of falls in the elderly by location. Aging Clin Exp Res 15:43-50

9. Bergland A, Wyller TB (2004) Risk factors for serious fall related injury in elderly women living at home. J Inj Prev 10:308-313

10. Boisgontier MP, Beets IA, Duysens J, Nieuwboer A, Krampe RT, Swinnen SP (2013) Age-related differences in attentional cost associated with postural dual tasks: increased recruitment of generic cognitive resources in older adults. J Neurosci Biobehav Rev 37:1824-1837

11. Botolfsen P, Helbostad JL, Moe-nilssen R, Wall JC (2008) Reliability and concurrent validity of the Expanded Timed Up-and-Go test in older people with impaired mobility. Physiother Res Int 13:94-106

12. Cesari M, Landi F, Torre S, Onder G, Lattanzio F, Bernabei R (2002) Prevalence and risk factors for falls in an older community-dwelling population. J Gerontol A Biol Sci Med Sci 57:M722-M726 
13. Chandler JM, Hadley EC (1996) Exercise to improve physiologic and functional performance in old age. J Clin Geriatr Med 12:761-784

14. Davatgaran K, Moghadam M, Salavati M (2009) The reliability of postural balance measures in single and dual tasking in healthy elders. J Iranian Journal of Ageing 4:17-25

15. De Souto BP, Morley JE, Chodzko-Zajko W, Pitkala KH, Weening-Djiksterhuis E, Rodriguez-Mañas L et al (2016) Recommendations on physical activity and exercise for older adults living in long-term care facilities: a taskforce report. J Am Med Dir Assoc 17:381-392

16. Długosz-Boś M, Filar-Mierzwa K, Stawarz R, Ścisłowska-Czarnecka A, Jankowicz-Szymańska A, Bac A (2021) Effect of three months pilates training on balance and fall risk in older women. Int J Environ Res Public Health 18:3663

17. Downs S, Marquez J, Chiarelli P (2013) The Berg Balance Scale has high intra-and inter-rater reliability but absolute reliability varies across the scale: a systematic review. J Physiother 59:93-99

18. Eston R, Reilly T. Kinanthropometry and exercise physiology laboratory manual: tests, procedures and data: volume two: physiology. 3, revised ed: Routledge; 2013.

19. Falbo S, Condello G, Capranica L, Forte R, Pesce C (2016) Effects of physical-cognitive dual task training on executive function and gait performance in older adults: a randomized controlled trial. Biomed Res Int 2016:5812092

20. Gharaeipour M, Andrew MK (2013) Examining Cognitive Status of elderly iranians: farsi version of the modified mini-mental state examination. Appl Neuropsychol Adult 20:215-220

21. Gillespie LD, Robertson MC, Gillespie WJ, Sherrington C, Gates S, Clemson $L$ et al (2012) Interventions for preventing falls in older people living in the community. Cochrane Database Syst Rev 2012(9):CD007146

22. Giorgetti MM, Harris BA, Jette A (1998) Reliability of clinical balance outcome measures in the elderly. Physiother Res Int 3:274-283

23. Gómez JF, Curcio C-L, Alvarado B, Zunzunegui MV, Guralnik J (2013) Validity and reliability of the Short Physical Performance Battery (SPPB): a pilot study on mobility in the Colombian Andes. Colomb Med 44:165-171

24. Guralnik JM, Ferrucci L, Pieper CF, Leveille SG, Markides KS, Ostir GV et al (2000) Lower extremity function and subsequent disability: consistency across studies, predictive models, and value of gait speed alone compared with the short physical performance battery. J Gerontol A Biol Sci Med Sci 55:M221-M231

25. lersel MBV, Kessels RP, Bloem BR, Verbeek AL, OldeRikkert MG (2008) Executive functions are associated with gait and balance in community-living elderly people. J Gerontol A Biol Sci Med Sci 63:1344-1349

26. Kannus P, Sievänen H, Palvanen M, Järvinen T, Parkkari J (2005) Prevention of falls and consequent injuries in elderly people. The Lancet 366:1885-1893

27. Koyama S, Tanabe S, Itoh N, Saitoh E, Takeda K, Hirano S et al (2018) Intra-and inter-rater reliability and validity of the tandem gait test for the assessment of dynamic gait balance. J European Journal of Physiotherapy 20:135-140

28. Lesinski M, Hortobágyi T, Muehlbauer T, Gollhofer A, Granacher U (2015) Effects of balance training on balance performance in healthy older adults: a systematic review and meta-analysis. Sports Med 45:1721-1738

29. Li KZ, Bherer L, Mirelman A, Maidan I, Hausdorff JM (2018) Cognitive involvement in balance, gait and dual-tasking in aging: a focused review from a neuroscience of aging perspective. Front Neurol 9:913

30. Lundebjerg N, Rubenstein L, Kenny R, Koval K, Martin F, Tinetti M et al (2001) Guideline for the prevention of falls in older persons. J Am Geriatr Soc 49:664-672

31. Mathias S, Nayak US, Isaacs B (1986) Balance in elderly patients: the" getup and go" test. Arch phys Med Rehabil 67:387-389

32. Muhaidat J, Kerr A, Evans JJ, Pilling M, Skelton DA (2014) Validity of simple gait-related dual-task tests in predicting falls in community-dwelling older adults. J Arch Phys Med 95:58-64

33. Pereira C, Rosado H, Cruz-Ferreira A, Marmeleira J (2018) Effects of a 10-week multimodal exercise program on physical and cognitive function of nursing home residents: a psychomotor intervention pilot study. Aging Clin Exp Res 30:471-479

34. Plummer P, Zukowski LA, Giuliani C, Hall AM, Zurakowski D (2016) Effects of physical exercise interventions on gait-related dual-task interference in older adults: a systematic review and meta-analysis. Gerontol 62:94-117
35. Rezola-Pardo C, Arrieta H, Gil SM, Yanguas JJ, Iturburu M, Irazusta J et al (2019) A randomized controlled trial protocol to test the efficacy of a dual-task multicomponent exercise program in the attenuation of frailty in long-term nursing home residents: Aging-on Dual-task study. J BMC Geriatr 19:6

36. Rodriguez-Larrad A, Arrieta H, Rezola C, Kortajarena M, Yanguas JJ, Iturburu M et al (2017) Effectiveness of a multicomponent exercise program in the attenuation of frailty in long-term nursing home residents: study protocol for a randomized clinical controlled trial. J BMC Geriatr 17:60

37. Schaefer S, Schumacher V (2011) The interplay between cognitive and motor functioning in healthy older adults: findings from dual-task studies and suggestions for intervention. Gerontology 57:239-246

38. Sedaghati P, Goudarzian M, Daneshmandi H, Ardjmand A (2018) Effects of Alexander-based corrective techniques on forward flexed posture, risk of fall, and fear of falling in idiopathic Parkinson's disease. J Arch Neurosci 5:e61274

39. Shumway-Cook A, Silver IF, LeMier M, York S, Cummings P, Koepsell TD (2007) Effectiveness of a community-based multifactorial intervention on falls and fall risk factors in community-living older adults: a randomized, controlled trial. J Gerontol A Biol Sci Med Sci 62:1420-1427

40. Springer S, Giladi N, Peretz C, Yogev G, Simon ES, Hausdorff JM (2006) Dual-tasking effects on gait variability: The role of aging, falls, and executive function. Mov Disord 21:950-957

41. Stins JF, Beek PJ (2012) A critical evaluation of the cognitive penetrability of posture. J Exp Aging Res 38:208-219

42. Stuss DT, Levine B (2002) Adult clinical neuropsychology: lessons from studies of the frontal lobes. J Annu Rev Psychol 53:401-433

43. Tabatabai AsI S, Sedaghati P (2021) The effect of combining CawthorneCooksey and Frankel exercises on functional balance and Fall probability in the elderly people with a history of falls. Scientific Journal of Rehabilitation Medicine 10: https://doi.org/10.22037/JRM.22021.114843.112614

44. Tinetti ME, Kumar C (2010) The patient who falls:"It's always a trade-off." JAMA 303:258-266

45. Van Impe A, Bruijn SM, Coxon JP, Wenderoth N, Sunaert S, Duysens J et al (2013) Age-related neural correlates of cognitive task performance under increased postural load. J Age 35:2111-2124

46. World Health Organization. Regional Committee for Europe 66th Session. Action plan for the prevention and control of noncommunicable diseases in the WHO European Region. http://www.euro.who.int/_data/ assets/pdf_file/0011/315398/66wd11e_NCDActionPlan_160522.pdf? ua =1. Copenhagen, Denmark, 2016. http://www.euro.who.int/_data/ assets/pdf_file/0011/315398/66wd111e_NCDActionPlan_160522.pdf? ua=1.Accesed 21 Feb 2017

47. Zijlstra A, Ufkes T, Skelton DA, Lundin-Olsson L, Zijlstra W (2008) Do dual tasks have an added value over single tasks for balance assessment in fall prevention programs? A mini-review. J Gerontology 54:40-49

\section{Publisher's Note}

Springer Nature remains neutral with regard to jurisdictional claims in published maps and institutional affiliations.

\section{Submit your manuscript to a SpringerOpen ${ }^{\circ}$ journal and benefit from:}

- Convenient online submission

- Rigorous peer review

- Open access: articles freely available online

- High visibility within the field

- Retaining the copyright to your article

Submit your next manuscript at springeropen.com 